\title{
DECAY OF WALSH SERIES AND DYADIC DIFFERENTIATION
}

BY

\author{
WILLIAM R. WADE
}

\begin{abstract}
Let $W_{2} n[f]$ denote the $2^{n}$ th partial sums of the Walsh-Fourier series of an integrable function $f$. Let $\rho_{n}(x)$ represent the ratio $W_{2} n[f, x] / 2^{n}$, for $x \in[0,1]$, and let $T(f)$ represent the function $\left(\sum \rho_{n}^{2}\right)^{1 / 2}$. We prove that $T(f)$ belongs to $L^{p}[0,1]$ for all $0<p<\infty$. We observe, using inequalities of Paley and Sunouchi, that the operator $f \rightarrow T(f)$ arises naturally in connection with dyadic differentiation. Namely, if $f$ is strongly dyadically differentiable (with derivative $\dot{D} f$ ) and has average zero on the interval $[0,1]$, then the $L^{p}$ norms of $f$ and $T(\dot{D} f)$ are equivalent when $1<p<\infty$. We improve inequalities implicit in Sunouchi's work for the case $p=1$ and indicate how they can be used to estimate the $L^{1}$ norm of $T(\dot{D} f)$ and the dyadic $H^{1}$ norm of $f$ by means of mixed norms of certain random Walsh series. An application of these estimates establishes that if $f$ is strongly dyadically differentiable in dyadic $H^{1}$, then $\int_{0}^{1} \Sigma_{N=1}^{\infty} \mid W_{N}[f, x]-\sigma_{N}[f, x] / N d x<\infty$.
\end{abstract}

1. Introduction. Let $m$ represent Lebesgue measure on the unit interval $[0,1]$. Let $w_{0}, w_{1}, \ldots$ represent the Walsh functions (Paley's ordering) and let $r_{0}, r_{1}, \ldots$ represent the Rademacher functions, i.e., $r_{k}=w_{2} k$ for $k=0,1, \ldots$ (see [3 or 7]).

Let

$$
W \equiv \sum_{k=0}^{\infty} a_{k} w_{k}
$$

be a Walsh series. The partial sums $\sum_{k=0}^{N-1} a_{k} w_{k}$ will be denoted by $W_{N}$, and the Cesaro means $\sum_{k=0}^{N-1}(1-k / N) a_{k} w_{k}$ will be denoted by $\sigma_{N}, N=1,2, \ldots$. For each integer $n \geqslant 0$, the dyadic block $W_{2^{n+1}}-W_{2^{n}}$ will be denoted by $\Delta_{n}(W)$.

The square function associated with (1) is

$$
S(W)=\left(\sum_{n=0}^{\infty} \Delta_{n}^{2}(W)\right)^{1 / 2},
$$

and the trave function associated with (1) is

$$
T(W)=\left(\sum_{n=0}^{\infty}\left(2^{-n} W_{2^{n}}\right)^{2}\right)^{1 / 2} .
$$

Finally, the formal dyadic derivative of (1) is the series

$$
\dot{W} \equiv \sum_{k=0}^{\infty} k a_{k} w_{k}
$$

Received by the editors April 19, 1982.

1980 Mathematics Subject Classification. Primary 42C10; Secondary 43A75.

Key words and phrases. Walsh series, dyadic derivative, square function, maximal function, dyadic $H^{1}$. 
Observe once and for all that

$$
\dot{W}_{N}(x)=N\left(W_{N}(x)-\sigma_{N}(x)\right)
$$

holds for every $x \in[0,1]$ and for each integer $N \geqslant 1$.

Let $W[f]$ denote the Walsh-Fourier series of a function $f \in L^{1}[0,1]$, i.e. $W[f] \equiv$ $\sum_{k=0}^{\infty} a_{k}(f) w_{k}$, where for each integer $k \geqslant 0, a_{k}(f)=\int_{0}^{1} f(x) w_{k}(x) d x$. The corresponding partial sums, Cesaro means, dyadic blocks, square function, trave function, and formal dyadic derivative will be denoted by $W_{N}[f], \sigma_{N}[f], \Delta_{n}(f), S(f), T(f)$, and $\dot{W}[f]$.

Recall that an integrable function $f$ is said to belong to dyadic $H^{1}$ if $S(f)$ is integrable. In such a case, the $H^{1}$ norm of $f$ is defined by

$$
\|f\|_{H^{1}}=\|S(f)\|_{L^{1}} \text {. }
$$

This norm turns out to be equivalent (see [4]) to $\left\|f^{*}\right\|_{L^{1}}$ where $f^{*} \equiv \sup _{n>0}\left|W_{2^{n}}[f]\right|$ represents the maximal function associated with $f$. Moreover, if $p>1$ then

$$
(1 / \sqrt{2})\|f\|_{L^{1}} \leqslant\|f\|_{H^{1}} \leqslant(p /(p-1))\|f\|_{L^{p}},
$$

and

$$
\left\|f^{*}\right\|_{L^{p}} \leqslant 10 p\|f\|_{L^{p}}
$$

Thus the role of the square function is to provide a space intermediate between the spaces $L^{p}[0,1], p>1$, and the space $L^{1}[0,1]$.

What is the role of the trave function? It provides a method of measuring the decay of $2^{-n} W_{2^{n}}$. Indeed, if $T(f, x)$ is finite then $\left\{2^{-n} W_{2^{n}}[f, x]\right\}_{n=0}^{\infty}$ converges to zero rather rapidly since it necessarily belongs to $l^{2}$. This convergence is faster than what is normally expected since a given integrable function satisfies $2^{-n} W_{2^{n}}[f] \rightarrow 0$ uniformly, as $n \rightarrow \infty$, but there are examples to show that this convergence can be as slow as one wishes.

It comes as somewhat of a surprise, then, that $T(f)$ is a.e. finite on $[0,1]$ when $f$ belongs to dyadic $H^{1}$. In fact, since the definition of $T(f)$ implies that $T(f) \leqslant f^{*}$. $T(f)$ actually belongs to $L^{1}[0,1]$ when $f$ belongs to dyadic $H^{1}$.

The main purpose of this paper is to show much more, namely the unexpected fact that $T(f) \in L^{p}[0,1]$ for all $0<p<\infty$ when $f \in L^{1}[0,1]$. Indeed, in $\$ 2$ we shall prove the following theorem.

THEOREM 1. If $2 \leqslant p<\infty$ then

$$
\|T(f)\|_{p} \leqslant(\sqrt[p]{4} /(\sqrt[p]{4}-1))^{1 / 2} \cdot\|f\|_{1} .
$$

A secondary purpose of this paper is to begin to study what happens to Sunouchi's inequalities [8] when $p=1$. In $\$ 3$ we shall obtain bounds for the $L^{1}$ norms of $T(\dot{W}), S(W)$ and of the function

$$
U(W)=\left(\sum_{N=1}^{\infty}\left|W_{N}-\sigma_{N}\right|^{2} / N\right)^{1 / 2}
$$


which improve those implicit in [8]. The most important of these is the bound for $T(\dot{W})$, and our method for obtaining this bound requires that $T(\dot{W})$ belongs to $L^{p}[0,1]$ for some $p>1$. In view of Theorem 1 , this will always be the case if $\dot{W}$ is a Walsh-Fourier series.

We use this observation in $\S 4$ to apply the results of $\$ 3$ to dyadically differentiable functions.

2. A proof of Theorem 1. Let $r=p / 2$ and observe by the Minkowski inequality that

$$
\|T(f)\|_{p}^{2} \equiv\|T(f)\|_{2 r}^{2} \leqslant \sum_{n=0}^{\infty} 2^{-2 n}\left\|W_{2^{n}}^{2}[f]\right\|_{r} .
$$

In particular,

$$
\|T(f)\|_{p} \leqslant\left(\sum_{n=0}^{\infty} 2^{-2 n}\left\|W_{2^{n}}[f]\right\|_{p}^{2}\right)^{1 / 2}
$$

holds for any $p \geqslant 2$ and any $f \in L^{1}[0,1]$.

Next, for each integer $n \geqslant 0$ use dyadic convolution to write $W_{2^{n}}[f]$ as $D_{2^{n}} * f$, where $D_{2^{n}}$ represents the $2^{n}$ th partia! sums of the Walsh-Dirichlet kernel. It follows from the Fubini theorem that

$$
\left\|W_{2^{n}}[f]\right\|_{p} \leqslant\|f\|_{1}\left\|D_{2^{n}}\right\|_{p}
$$

holds for $p \geqslant 1, n \geqslant 0$. However, it is well known (see [3]) that $D_{2^{n}}(x)=2^{n}$ if $0 \leqslant x<2^{-n}$ and $D_{2^{n}}(x)=0$ for $2^{-n} \leqslant x \leqslant 1$. In particular, (10) implies that

$$
\left\|W_{2^{n}}[f]\right\|_{p} \leqslant\|f\|_{1} 2^{n(1-1 / p)}
$$

holds for all $p \geqslant 1$ and $n \geqslant 0$. Finally, if we apply inequality (11) to the appropriate expression on the right side of (9), we conclude that

$$
\|T(f)\|_{p} \leqslant\left(\sum_{n=0}^{\infty} 2^{-2 n} 2^{2 n(1-1 / p)}\|f\|_{1}^{2}\right)^{1 / 2} \equiv\|f\|_{1}\left(\sum_{n=0}^{\infty} 2^{-2 n / p}\right)^{1 / 2} .
$$

The proof of Theorem 1 is complete.

3. Estimates for $S, T$ and $U$. In view of (5) the main result in [8] can be summarized as follows.

TheOREm A. Let $1<p<\infty$. There exist constants $E_{p}, F_{p}$ and $G_{p}$ such that

$$
\|U(f)\|_{L^{p}} \leqslant E_{p}\|T(\dot{W}[f])\|_{L^{p}} \leqslant F_{p}\|S(f)\|_{L^{p}} \leqslant G_{p}\|U(f)\|_{L^{p}}
$$

holds for every $f \in L^{p}[0,1]$ with $a_{0}(f)=0$.

Since $H^{1}$ is a proper subset of $L^{1}$, Theorem A cannot hold when $p=1$. Theorem A does contain estimates for $L^{1}$ norms of $U(f), T(\dot{W}[f])$, and $S(f)$. For example, since $\|g\|_{L^{1}} \leqslant\|g\|_{L^{p}}$ holds for $p>1$ and for any measurable $g$, we have that

$$
\|U(f)\|_{L^{1}} \leqslant E_{p}\|T(\dot{W}[f])\|_{L^{p}}
$$

holds for any $p>1$. 
In this section we derive inequalities for certain Walsh series which when specialized to the Walsh-Fourier series case offer improvements to the estimates above. Before stating our results we need addtional notation.

Let $W$ be a Walsh series, let $D$ be the Walsh-Dirichlet kernel, and let $(x, \theta)$ be any point in the unit cube $Q$. Set

$$
\begin{aligned}
& T W(x, \theta)=\sum_{n=0}^{\infty} r_{n}(\theta) \sum_{k=2^{n}}^{2^{n+1}-1} \frac{1}{k^{3}} w_{k}(x) W_{2^{n}}(x) \\
& S W(x, \theta)=\sum_{n=0}^{\infty} r_{n}(\theta) \frac{1}{2^{n}} \sum_{i=0}^{n-1}\left(W_{2^{n}}-W_{2^{i}}\right)\left(D_{2^{i+1}}-D_{2^{i}}\right)(x)
\end{aligned}
$$

and

$$
U W(x, \theta)=\sum_{n=1}^{\infty} r_{n}(\theta) w_{n}(x) \frac{W_{n}(x)-\sigma_{n}(x)}{\sqrt{n}} .
$$

We shall prove the following result.

Theorem 2. Suppose that $W$ is a Walsh series with no constant term. There is an absolute constant $A$, independent of $W$, such that:

(i) if $T(\dot{W}) \in L^{p}[0,1]$ for some $p>1$ then

$$
\|U(W)\|_{L^{1}} \leqslant A \int_{0}^{1}\|T \dot{W}(\cdot, \theta)\|_{H^{1}} d \theta ;
$$

(ii) if $S(W) \in L^{p}[0,1]$ for some $p>1$ then

$$
\|T(\dot{W})\|_{L^{1}} \leqslant A \int_{0}^{1}\|S W(\cdot, \theta)\|_{H^{1}} d \theta
$$

and,

(iii) if $U(W) \in L^{p}[0,1]$ for some $p>1$ then

$$
\|S(W)\|_{L^{1}} \leqslant A \int_{0}^{1}\|U W(\cdot, \theta)\|_{H^{1}} d \theta+2\|U(W)\|_{L^{1}} .
$$

We first show that these estimates improve those implicit in Theorem A.

LEMMA 2. For each $p>1$ there exists a constant $B_{p}$ such that

$$
\begin{aligned}
& \int_{0}^{1}\|T \dot{W}(\cdot, \theta)\|_{H^{1}} d \theta \leqslant B_{p}\|T(\dot{W})\|_{L^{p}}, \\
& \int_{0}^{1}\|S W(\cdot, \theta)\|_{H^{1}} d \theta \leqslant B_{p}\|S(W)\|_{L^{p}},
\end{aligned}
$$

and

$$
\int_{0}^{1}\|U W(\cdot, \theta)\|_{H^{1}} d \theta \leqslant B_{p}\|U(W)\|_{L^{p}}
$$

hold for all Walsh series $W$.

To verify (15) apply (7), Jensen's inequality and Fubini's theorem to conclude that

$$
\int_{0}^{1}\|T \dot{W}(\cdot, \theta)\|_{H^{1}} d \theta \leqslant \frac{p}{p-1}\left\{\int_{0}^{1} \int_{0}^{1}|T \dot{W}(x, \theta)|^{p} d \theta d x\right\}^{1 / p} .
$$


By Khinchin's inequality [10, p. 213] this last term is dominated by

$$
B_{p}\left\{\int_{0}^{1}\left[\sum_{n=0}^{\infty} \dot{W}_{2^{n}}^{2}(x) w_{n}^{2}(x)\left(\sum_{k=2^{n}}^{2^{n+1}-1} \frac{1}{k^{3}}\right)^{2}\right]^{p / 2} d x\right\}^{1 / p} .
$$

Since $w_{n}^{2}(x) \equiv 1$, the expression above is no greater than $B_{p}\|T(\dot{W})\|_{L^{p}}$, as required.

Similarly, the left-hand side of (16) is dominated by

$$
B_{p}\left\{\int_{0}^{1}\left[\sum_{n=0}^{\infty} \frac{1}{4^{n}}\left(\sum_{i=1}^{n}\left(W_{2^{n}}-W_{2^{i}}\right)\left(D_{2^{i+1}}-D_{2^{i}}\right)\right)^{2}\right]^{p / 2} d m\right\}^{1 / p} .
$$

But $D_{2^{m}}(x)$ is well known to be $2^{m}$ for $0 \leqslant x<2^{-m}$ and zero otherwise. Thus by Schwarz, we deduce that

$$
\left(\sum_{i=1}^{n}\left(W_{2^{n}}-W_{2^{i}}\right)\left(D_{2^{i+1}}-D_{2^{i}}\right)\right)^{2} \leqslant 2 n \sum_{i=1}^{n} 2^{i}\left(W_{2^{n}}-W_{2^{i}}\right)^{2} .
$$

It follows that

$$
\int_{0}^{1}\|S W(\cdot, \theta)\|_{H^{1}} d \theta \leqslant B_{p}\left\{\int_{0}^{1}\left[\sum_{n=0}^{\infty} \frac{1}{2^{n}} \sum_{i=1}^{n} 2^{i}\left(W_{2^{n}}-W_{2^{i}}\right)^{2}\right]^{p / 2} d m\right\}^{1 / p} .
$$

This last term can be shown to be no greater than $B_{p}\|S(W)\|_{L^{p}}$ by repeating the steps indicated in the middle of p. 195 in [9].

A similar argument establishes (17).

The following is the main lemma of this section.

LEMMA 3. If $\left\{f_{k}\right\}_{k=0}^{\infty}$ is a sequence of functions which satisfies

$$
\int_{0}^{1}\left(\sum_{k=0}^{\infty} f_{k}^{2}(x)\right)^{p / 2} d x<\infty \text { for some } p>1
$$

then

$$
\int_{0}^{1}\left(\sum_{k, n=0}^{\infty} \Delta_{n}^{2}\left(f_{k}\right)\right)^{1 / 2} d m \leqslant C \int_{0}^{1}\left\|\sum_{n=0}^{\infty} r_{n}(\theta) f_{n}\right\|_{H^{1}} d \theta
$$

where $C$ is an absolute constant which does not depend on the functions $f_{k}$.

To prove this lemma set

$$
F_{k}(x, \phi)=\sum_{n=0}^{\infty} r_{n}(\phi) \Delta_{n}\left(f_{k}, x\right)
$$

and

$$
\Psi(x, \phi, \theta)=\sum_{k=0}^{\infty} r_{k}(\theta) F_{k}(x, \phi)
$$


for $x, \phi, \theta \in[0,1]$. Recall by the two-dimensional version of Khinchin's inequality $\left[6\right.$, p. 84] that there are constants $C^{\prime}$ and $C^{\prime \prime}$ such that

$$
\begin{aligned}
C^{\prime}\left(\sum_{n, k=0}^{\infty} \Delta_{n}^{2}\left(f_{k}, x\right)\right)^{1 / 2} & \leqslant \iint_{Q}|\Psi(x, \phi, \theta)| d m(\phi, \theta) \\
& \leqslant C^{\prime \prime}\left(\sum_{n, k=0}^{\infty} \Delta_{n}^{2}\left(f_{k}, x\right)\right)^{1 / 2}
\end{aligned}
$$

By the left-hand side of (20), together with (7) and Fubini's theorem we have that

$$
\int_{0}^{1}\left(\sum_{n, k=0}^{\infty} \Delta_{n}^{2}\left(f_{k}\right)\right)^{1 / 2} d m \leqslant C \iint_{Q}\|\Psi(\cdot, \phi, \theta)\|_{H^{\prime}} d m(\phi, \theta)
$$

where $C=\sqrt{2} / C^{\prime}$. By the right-hand side of (20) and Lemma 2 of [8] we conclude that

$$
\int_{0}^{1} \int_{0}^{1} \int_{0}^{1}|\Psi(x, \phi, \theta)| d x d \phi d \theta \leqslant C^{\prime \prime \prime} \int_{0}^{1}\left(\sum_{k=0}^{\infty} f_{k}^{2}\right)^{p / 2} d m
$$

holds for any $p>1$. By (18), then, the series $\Psi$ converges in $L^{1}[0,1]$ norm for a.e. $(\phi, \theta) \in Q$. In particular, $\Psi$ is a Walsh-Fourier series for almost every $(\theta, \phi) \in Q$. A similar argument establishes that $\sum_{k=0}^{\infty} r_{k}(\theta) f_{k}$ is also a Walsh-Fourier series for a.e. $\theta \in[0,1]$. Since the Walsh-Fourier coefficients of $F_{k}(\cdot, \phi)$ and $f_{k}$ are identical, we conclude that

$$
\left\|\sum_{k=0}^{\infty} r_{k}(\theta) f_{k}\right\|_{H^{1}} \equiv\|\Psi(\cdot, \phi, \theta)\|_{H^{1}}
$$

holds for a.e. $\phi$ and $\theta$ in the interval $[0,1]$. The proof of Lemma 3 is completed by applying this norm equality to the right-hand side of (21).

By repeating the argument found in [8, pp. 7-8] one can obtain a slight extension of Lemma 3: if $\left\{f_{k}\right\}_{k=0}^{\infty}$ is a sequence of functions satisfying (18) for some $p>1$ and if $\left\{q_{k}\right\}_{k=0}^{\infty}$ is a sequence of positive integers diverging to $+\infty$, then

$$
\int_{0}^{1}\left(\sum_{k=0}^{\infty}\left|W_{q_{k}}\left(f_{k}\right)\right|^{2}\right)^{1 / 2} d m \leqslant \sqrt{2} C \int_{0}^{1}\left\|\sum_{k=0}^{\infty} r_{k}(\theta) w_{q_{k}} f_{k}\right\|_{H^{1}} d \theta \text {. }
$$

A sketch of this argument is that (7) is used to show the left-hand side of (22) is dominated by $\sqrt{2} \int_{0}^{1} \sqrt{\sum_{n, k=0}^{\infty} \Delta_{n}^{2}\left(w_{q_{k}} f_{k}\right)} d m$, and then (22) is established by a single application of Lemma 3.

The proof of Theorem 2 consists of three applications of inequality (22). For (i) use (5) to write $U^{2}(W)$ as

$$
\sum_{N=1}^{\infty}\left|\frac{\dot{W}_{N}}{N^{3}}\right|^{2} \equiv \sum_{n=1}^{\infty} \sum_{k=2^{n-1}}^{2^{n}-1}\left|W_{k}\left(\frac{\dot{W}_{2^{n}}}{k^{3}}\right)\right|^{2}
$$

Thus (12) follows from (22) with $q_{k} \equiv k$ and $f_{k}=\dot{W}_{2^{n}} / k^{3}$ where $k$ and $n$ are related by $2^{n-1} \leqslant k<2^{n}$. Observe that (18) follows from the hypothesis that $T(f) \in L^{p}[0,1]$ for some $p>1$. 
For (ii) repeat the argument occurring at the bottom of p. 194 in [9] to conclude that

$$
T(\dot{W}) \leqslant \sum_{n=1}^{\infty} 2^{-n} \sum_{k=0}^{2^{n}}\left|W_{2^{n}}-W_{k}\right|^{2}
$$

If we note that (22) applies not only to partial sums but to any connected block of terms of Walsh series (such blocks are differences of two partial sums), we obtain (13) from (23) as in [9].

Finally, for (iii) we use a string of inequalities which appears at the top of p. 10 in [8] to see that

$$
\|S(W)\|_{L^{1}} \leqslant 2\|U(W)\|_{L^{1}}+2 \int_{0}^{1}\left\{\sum_{n=1}^{\infty} \frac{\dot{W}_{n}^{2}}{n^{3}}\right\}^{1 / 2} d m .
$$

A final application of (22) followed by (5) establishes (14) and thus completes the proof of Theorem 2 .

4. Applications to the dyadic derivative. In this section we apply Theorems A and 2 of $\S 3$ to obtain inequalities relating a function $f$ to its dyadic derivative $D f$.

Recall that a function $f$ defined on $[0,1]$ is said to be dyadically differentiable in $L^{p}$ for some $p \geqslant 1$ if the sequence.

$$
d_{N}(f, x) \equiv \sum_{k=0}^{N-1} 2^{k-1}\left[f(x)-f\left(x+2^{-k-1}\right)\right], \quad x \in[0,1],
$$

converges in the $L^{p}$ norm, as $N \rightarrow \infty$. In such a case the limit, denoted by $\dot{D} f$, is called the (strong) dyadic derivative of $f$. Since $\dot{D} w_{k}=k w_{k}$ for any integer $k \geqslant 0$, the formal dyadic derivative $\dot{W}$ is simply the term by term dyadic derivative of the Walsh series $W$. Butzer and Wagner [1] have shown that an integrable function $f$ is dyadically differentiable in $L^{p}$, for some $p \geqslant 1$, if and only if $\dot{W}[f]$ is the WalshFourier series of some $g \in L^{p}[0,1]$, in which case $\dot{D} f=g$ a.e. A similar definition and result holds for $H^{1}$ as well [5]. Thus, if $f$ is dyadically differentiable in the strong sense, then $\dot{W}[f]=W[\dot{D} f]$.

For any $p \geqslant 1$ let $\mathscr{W}$ denote these integrable functions $h$ which satisfy $T(h) \in$ $L^{p}[0,1]$, and set $\|h\|_{\mho_{p}}=\|T(h)\|_{L^{p}}$. If we deal only with those functions $h \in \mathcal{Q} \int_{p}$ which satisfy $a_{0}(h)=0$, then $\mho_{p}$ is a normed linear space. By Holder's inequality, the $\mho_{p}$ norms get weaker as $p$ get smaller. Moreover, by Theorem 1 the $\mho_{p}$ norms are all weaker than the $L^{1}$ norm, $p \neq \infty$. However, no $\mathscr{W}_{p}$ is complete.

The following theorem is an easy consequence of Theorem A and Paley's inequality [7].

THEOREM 3. Let $f \in L^{1}[0,1]$ be dyadically differentiable in the strong sense and suppose that $a_{0}(f)=0$. If $1<p<\infty$ then there exists constants $\alpha_{p}$ and $\beta_{p}$ such that

$$
\alpha_{p}\|f\|_{L^{p}} \leqslant\|\dot{D} f\|_{\mho_{p}} \leqslant \beta_{p}\|f\|_{L^{p}} .
$$

This result contains an interesting corollary. If $f, f_{1}, f_{2}, \ldots$ is a sequence of dyadically differentiable functions then a necessary and sufficient condition for 
$f_{n} \rightarrow f$ in $L^{p}$ norm, as $n \rightarrow \infty$, for some $1<p<\infty$ is that $\dot{D} f_{n} \rightarrow \dot{D} f$ in $\mathcal{W f}_{p}$ norm, as $n \rightarrow \infty$. In particular, if $\dot{D} f_{n} \rightarrow \dot{D} f$ in $L^{1}$ norm, as $n \rightarrow \infty$, then $f_{n} \rightarrow f$ in $L^{p}$ norm, as $n \rightarrow \infty$, for every $p \neq \infty$.

Theorem 3 does not hold for $p=1$. Nevertheless, certain one-sided inequalities for $p=1$ can be derived from Theorem 2 by applying it to $W=W[f]$, where $f$ is dyadically differentiable in $L^{1}$, and those one-sided inequalities improve those implicit in Theorem 3 just as Theorem 2 improved those implicit in Theorem A. Moreover, each of the $H^{1}$ norms on the right side of inequalities (12), (13), and (14) can be computed exactly. Indeed, by the proof of Theorem 2 the series $T \dot{W}(\cdot, \theta)$, $S W(\cdot, \theta)$, and $U W(\cdot, \theta)$ all converge in $L^{1}$ norm, for a.e. $\theta \in[0,1]$. Hence the Walsh-Fourier coefficients of these series can be computed by orthogonality. We shall systematically investigate the resulting inequalities in a later paper. One is the interesting

$$
\|U(W[f])\|_{L^{2}} \leqslant \sqrt{2}\|\dot{D} f\|_{H^{1}},
$$

which holds for any dyadically differentiable $f$. Hence, if $f$ is dyadically differentiable in $H^{1}$, then

$$
\sum_{N=1}^{\infty} \int_{0}^{1} N^{-1}\left|W_{N}(f, x)-\sigma_{N}(f, x)\right| d x<\infty .
$$

\section{REFERENCES}

1. P. L. Butzer and H. J. Wagner, Walsh series and the concept of a derivative, Applicable Anal. 3 (1973), 29-46.

2. On dyadic analysis based on the pointwise dyadic derivative, Anal. Math. 1 (1975), 171-196.

3. N. J. Fine, On the Walsh functions, Trans. Amer. Math. Soc. 65 (1949), 372-414.

4. A. M. Garsia, Martingale inequalities, Benjamin, Reading, Mass., 1973.

5. N. R. Ladhawala, Absolute summability of Walsh-Fourier series, Pacific J. Math. 65 (1976), 103-108.

6. J. Marcinkiewicz, Sur les multiplicateurs des séries de Fourier, Studia Math. 8 (1939), 79-91.

7. R. E. A. C. Paley, A remarcable series of orthogonal functions. I, Proc. London Math. Soc. 34 (1931), 241-264.

8. G. I. Sunouchi, On the Walsh-Kaczmarz series, Proc. Amer. Math. Soc. 2 (1951), 5-11.

9. A. Zygmund, On the convergence and summability of power series in the circle of convergence. I, Fund. Math. 30 (1938), 170-196.

10. __ Trigonometric series, Vol. I, Cambridge Univ. Press, Cambridge, 1959.

Department of Mathematics, University of Tennessee, Knoxville, Tennessee 37916 